\title{
Thymectomy in myasthenia gravis during pregnancy
}

\author{
Mary S.M. Ip*, S.Y. So, W.K. Lam, Lawrence C.H. Tang and C.K. Mok \\ Departments of Medicine, Obstetrics \& Gynaecology, and Surgery, University of Hong Kong, Hong Kong
}

\begin{abstract}
Summary: A 24 year old woman with myasthenia gravis suffered an antepartum exacerbation which failed to respond to increasing dosages of pyridostigmine. Thymectomy performed in the second trimester was followed by clinical improvement and full term vaginal delivery with no puerperal deterioration.
\end{abstract}

\section{Introduction}

The course of myasthenia gravis in pregnancy is unpredictable (Giwa-Osagie et al., 1981). Complete remission, clinical improvement, acute exacerbations or no change in myasthenic symptoms have been reported. Anticholinesterase drugs and steroid treatment are the mainstays of medical therapy during pregnancy (Plauche, 1979). Thymectomy in the management of a pregnant myasthenic patient is rarely indicated and has not been documented previously.

\section{Case report}

A 24 year old Chinese primigravid woman first presented with ptosis and weakness of the lower limbs 2 years earlier. Myasthenia gravis was diagnosed by the edrophonium test in which $10 \mathrm{mg}$ of edrophonium intravenously produced marked improvement of ptosis and muscle power while normal saline produced no response. Electromyography showed decremental response in muscle action potential on repetitive supramaximal stimulation of the ulnar nerve. Pyridostigmine (Mestinon) $120 \mathrm{mg}$ five times a day was administered with good control of her myasthenic symptoms until she was 8 weeks pregnant.

She then developed dysphagia and dysarthria, progressive proximal muscle weakness and mild respiratory muscle involvement. The dose of pyridostigmine was adjusted by repeated edrophonium test to $180 \mathrm{mg}$ five times a day but the patient remained weak. Transsternal thymectomy was performed at the 17th gestational week. Histological examination revealed thymic hyperplasia. Postoperatively, she required

*Correspondence and present address: M.S.M. Ip, M.B., B.S., M.R.C.P., Department of Respiratory Medicine, University of Edinburgh, City Hospital, Greenbank Drive, Edinburgh, EH10 5SB, UK.

Accepted: 28 November 1985 mechanical ventilation for 8 days. Pyridostigmine was re-introduced on the second postoperative day and the dosage was adjusted by repeated edrophonium tests. The postoperative course was complicated by collapse of the left lung on the fifth day because the endotracheal tube had entered the right bronchus. Bronchial lavage was done with re-expansion of the left lung in one day. She was weaned off the respirator on the seventh postoperative day with the endotracheal tube retained for 2 more days for aspiration of copious bronchial secretion. She was discharged home on the 17 th postoperative day, maintained on pyridostigmine $180 \mathrm{mg}$ six times a day.

She went into spontaneous labour at the 39th gestational week, delivering a male infant of $3,169 \mathrm{~g}$ by vacuum extraction for slight meconium-stained liquor. Mild neonatal myasthenia was detected, which responded well to pyridostigmine treatment. No postpartum deterioration of her myasthenia was observed and within 4 weeks of delivery, her condition stabilized while she returned to pre-pregnancy dosage of pyridostigmine of $120 \mathrm{mg}$ five times a day.

\section{Discussion}

Thymectomy has become established in the treatment of myasthenia gravis since it leads to significant clinical improvement, lower anticholinesterase requirement and complete remission in a proportion of cases (Buckingham et al., 1976). The risk of thymectomy has lessened with experience in the surgical techniques and facilities for intensive care. Study of the use of thymectomy in young, non-pregnant female myasthenic patients has demonstrated increased incidence of remission and decreased incidence of exacerbation during pregnancy in the thymectomy group (Eden \& Gall, 1983). However, there is no documented report of thymectomy during pregnancy 
for the control of myasthenic crisis. Our patient, who received pyridostigmine alone without steroid treatment, illustrates the beneficial outcome following midtrimester thymectomy. Thymectomy should be con-

\section{References}

BUCKINGHAM, J.M., HOWARD, F.M., BERNATZ, P.E., SPENCER PAYNE, W., HARRISON, E.G. JR., O'BRIEN, P.C. \& WEILAND, L.H. (1976). The value of thymectomy in myasthenia gravis. A computer matched study. Annals of Surgery, 184, 453.

EDEN, R.D. \& GALL, S.A. (1983). Myasthenia gravis and pregnancy: A reappraisal of thymectomy. Obstetrics and Gynecology, 62, 328. sidered in the management of deteriorating myasthenia gravis during pregnancy as an alternative to steroid therapy or plasmapheresis.

GIWA-OSAGIE, O.F., NEWTON, J.R. \& LARCHER, V. (1981). Obstetric performance of patients with myasthenia gravis. International Journal of Gynecology and Obstetrics, 19, 267.

PLAUCHE, W.C. (1979). Myasthenia gravis in pregnancy: an update. American Journal of Obstetrics and Gynecology, 135, 691. 